\title{
EXTOD: Exploring the barriers and benefits of physical exercise for people with type 1 diabetes
}

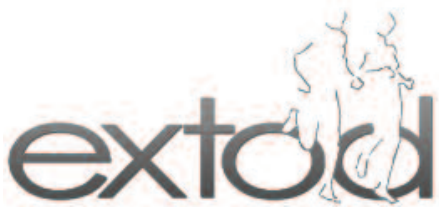

\author{
PARTH NARENDRAN, ${ }^{1,2}$ ROB C ANDREWS ${ }^{3,4}$
}

Key words: type 1 diabetes, exercise, beta cell, barriers, education, RCT

EXercise for Type One Diabetes (EXTOD) is a programme of work that aims to explore the barriers and benefits of physical exercise for people with type 1 diabetes (T1D). Its overarching aim is to provide evidence-based support for people with T1D to undertake safe and effective exercise, and to enjoy its associated health benefits. ${ }^{1}$ Here we outline the origins of EXTOD, a summary of what has been achieved so far, and a brief overview of future plans.

The first EXTOD study was a pilot study designed to explore whether the beta cell-preserving effects of exercise that have been demonstrated in people with type 2 diabetes and animal models of T1D also applied to patients with new-onset T1D. 2,3 This study was funded by the National Institute of Health Research (NIHR) and has been recently published. ${ }^{4}$ Being a pilot study, its primary aims were to address the key uncertainties in designing a definitive trial to test whether regular exercise preserves beta cells in people newly diagnosed with T1D. ${ }^{4}$ Fifty-eight adults with newly diagnosed T1D who had evidence of residual beta cell function were randomised to usual care or usual care with support to increase their levels of exercise to at least 150 min of moderate intensity exercise per week. The study showed that it was possible to recruit and randomise people with newly diagnosed T1D to a trial of an exercise intervention, and to increase and maintain their exercise levels for 12 months. However, only two-thirds of the exercise arm reached the target of $\geq 150 \mathrm{~min} /$ week. Furthermore, trial retention was challenging and $30 \%$ of the participants did not complete the study. In addition, this study showed for the first time that beta cell function, when corrected for the changes in insulin sensitivity that accompany physical exercise, may be preserved in people with T1D (Figure 1). This finding is supported by a case-control study that we carried

The Institute of Immunology and Immunotherapy, The Medical School, University of Birmingham, Birmingham, UK

Department of Diabetes, The Queen Elizabeth Hospital, Birmingham, UK

University of Exeter, Medical School, University of Exeter, Exeter, UK

4 Department of Diabetes, Taunton and Somerset NHS Foundation Trust, Taunton, UK

Address for correspondence: Dr Rob C Andrews

University of Exeter, Medical School, University of Exeter, Exeter, EX2 5DW, UK

Tel: +44 (0)1823 344536

E-mail: R.C.Andrews@exeter.ac.uk

Br J Diabetes 2018;18:97-99

https://doi.org/10.15277/bjd.2018.179
Figure 1. Beta cell function as represented by mean disposition index in participants from the control and intervention groups. Data from the EXTOD pilot study undertaken to explore whether exercise intervention delivered over one year preserves beta cell function in adults newly diagnosed with T1D

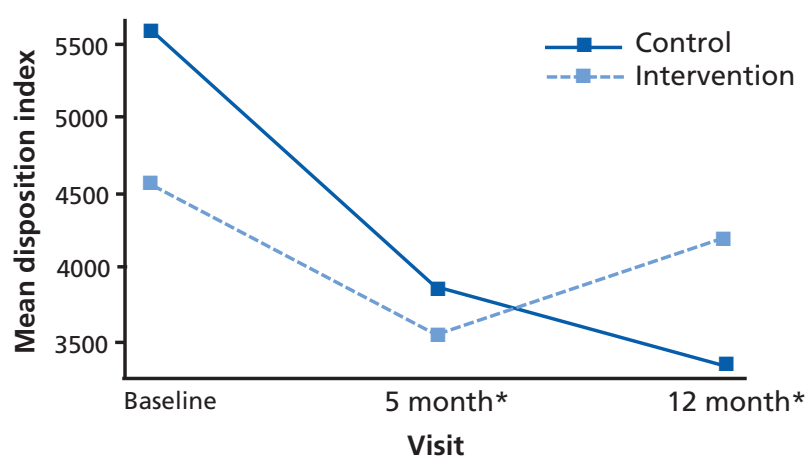

out in which the 'honeymoon' period appears to be five times longer in people who regularly exercise for more than 150 min per week. ${ }^{5}$

A home-based supervised exercise programme developed by collaborators in Liverpool has recently demonstrated adherence to exercise of over $90 \%$. Furthermore, qualitative interviews with participants from the EXTOD study have been conducted to understand how to improve retention rates in lifestyle trials with adults with new-onset T1D. ${ }^{6}$ This information will now be used to support a funding application for a formal randomised controlled trial (RCT) to explore whether physical exercise preserves residual beta cell function in adults with new-onset T1D. Within this study there will be sub-studies to understand more about how exercise affects beta cell function and viability.

The 'EXTOD Mechanisms' study was undertaken to explore the mechanisms through which physical exercise could preserve beta cell function in T1D. Early results suggest that exercise has the potential to modulate the behaviour of immune cells that target and destroy pancreatic beta cells (manuscripts in preparation). Peripheral blood samples were taken from people with T1D before and after a bout of vigorous exercise and the behaviour of immune cells in these samples was studied. Exercise modulated the ability of immune $T$ cells to migrate across endothelial cells in T1D. Transendothelial migration of $T$ cells is critical to the process by 
Table 1 Diabetes-specific barriers to exercise in adults with new-onset established T1D

\begin{tabular}{l|l}
\hline New-onset T1D & Established T1D \\
$\begin{array}{l}\text { - Hypoglycaemia (both actual } \\
\text { and fear of) }\end{array}$ & $\begin{array}{l}\text { - Loss of control of diabetes } \\
\text { - Lack of knowledge/confidence in } \\
\text { managing diabetes } \\
\text { - Advice from healthcare } \\
\text { professionals to stop exercising } \\
\text { when exercising }\end{array}$ \\
$\begin{array}{l}\text { - Planning (eg, checking } \\
\text { blood glucose) }\end{array}$ & \\
- Feeling overwhelmed by diagnosis &
\end{tabular}

which auto-reactive peripheral blood T cells access and destroy pancreatic beta cells, and modulating this process could therefore delay or even prevent T1D.

Significant qualitative work has been undertaken in the EXTOD programme to explore barriers to exercise in adults with established as well as in new-onset T1D. The barriers cited by adults with newonset T1D include fear of hypoglycaemia and the time required to plan for exercise (this included checking glucose levels and planning for exercise with regard to meals) (Table 1). ${ }^{7}$ Worryingly, some newly diagnosed adults had been advised not to exercise by their healthcare professional (HCP), and this advice had not been reversed after they had a chance to settle into their diagnosis and understand the basics of insulin management. Barriers cited by adults with established T1D include the effect of exercise disturbing their day-to-day diabetes control. ${ }^{8}$ Whilst many patients with established T1D were aware of the risks of hypoglycaemia, fear of hypoglycaemia was not specifically stated as a reason for not exercising. Adults in both studies cited lack of knowledge around managing diabetes when exercising as a barrier, and all overwhelmingly stated a desire for education on this subject. Simultaneous quantitative work with HCPs caring for people with T1D suggested that, although many HCPs thought they were competent in providing education on managing T1D during exercise, their knowledge levels in this area were poor when formally tested. Reassuringly, most HCPs wanted formal education in this area. ${ }^{9}$ These findings resulted in four main outcomes.

First, these findings resulted in the annual one-day EXTOD conference. This conference aims to equip HCPs with the knowledge to support their T1D patients to undertake exercise. The day includes plenary talks and workshops on the physiology of exercise, safety, supporting a patient with the complications of T1D to exercise, strategies to manage blood glucose before, during and after exercise. The talks and sessions are delivered by some of the most eminent international authorities in these areas. The conferences have been well received, always run to capacity, with over 650 participants since inception in 2014. Having initially started as the EXTOD conference, it has more recently expanded to partner with the JDRF and the ABCD. The 2018 conference will again be tri-partite and is scheduled for 19 October in Manchester (https://abcd.care/extod-peak/conference-2018).

Second, as a collaboration between the Universities of Exeter,
Birmingham and Leicester, the EXTOD Education programme has been developed. Funded by the NIHR, this programme is developing and piloting an education programme for people with T1D on multiple daily injections (MDI) (with accompanying training for HCPs to deliver this programme) to guide insulin and carbohydrate adjustment for safe exercise. The programme was developed using robust methodology based on the MRC framework for complex interventions, and by a multidisciplinary team consisting of experts in the field of exercise and T1D, and researchers with experience of developing self-management education. It was informed by a review of the evidence in T1D and exercise, behaviour change literature and qualitative interviews with stakeholders (manuscript in review). The programme and supporting resources were then refined using an iterative process of testing, delivery and collecting feedback from participants and the wider development team. ${ }^{10}$ This programme is now being tested in a pilot RCT. Funding is being sought to develop and test a similar programme for adults on insulin pump therapy, and then test both of these in a large RCT. Funding is also being sought to develop programmes for children on MDI and pumps, and to develop an online education course.

Third, whilst an education programme may help many people with T1D to undertake safe and effective exercise, there will always be a group of people who need a greater level of support for more challenging physical events. This understanding, and the recognition that there are already a number of people with T1D who have achieved significant sporting prowess, has led to a collaboration between ABCD and EXTOD to develop the 'EXTOD Buddies' programme. Here, an advocate with T1D who already has the skills and experience to undertake specified sporting or physical challenges will be paired with a person with T1D who wishes to undertake the same or similar activity. For example, a person planning to undertake a marathon run would be introduced to an advocate who has previously undertaken a marathon for ad hoc support and guidance. Each advocate would have been trained to ensure they are up to date with the required knowledge on practicalities and safety. The programme will be supported by online resources and forums.

Fourth, an understanding of the barriers to exercise - both medical and otherwise - has led to direct engagement with patients and the public to support sporting events that are encouraging and inspiring. Most recently, this has resulted in a collaboration with Paul Cocker (1bloodydrop.com) to support the Swansea Half Marathon in June 2018. Here, a world record was set when 69 people with T1D ran the half marathon. Many of the participants consented to participate in the 'EXTOD 101' research study, where data on their training programme and blood glucose variability measured by flash glucose sensing have been collected. This will, for the first time, provide a very valuable insight into how a large number of people with T1D approach training for extreme physical events, and the effect of such events on blood glucose. This understanding can be used to support others with T1D who wish to prepare for such events.

We have summarised the work undertaken by the EXTOD programme so far, and its longer term vision. As HCPs, it is our responsibility to recommend regular physical activity to our patients. ${ }^{11}$ 
While there is evidence that physical activity reduces heart disease and improves wellbeing in T1D, there are other benefits that need urgent exploration. Exploring these benefits may influence guidelines on when exercise should be initiated in the natural history of $\mathrm{T1D}$, and the intensity and duration of this exercise. Once exercise is recommended, it is important that patients can be given the right advice about how to manage their glucose around exercise and how to get the greatest benefit from their chosen activity. With continued support from clinical, academic and patient collaborators, EXTOD hopes over the next 5-10 years to make this knowledge available to everyone with T1D.

Conflict of interest RCA has received honoraria from Novo Nordisk, Sanofi-Aventis and Merck Sharp \& Dohme, and travel expenses from SanofiAventis. PN has received honoraria and travel expenses from Novo Nordisk, Astra Zeneca, Abbott and Lilly.

Funding Some of the work outlined in this report was funded by the National Institute for Health Research (NIHR).

Disclaimer The views expressed are those of the authors and not necessarily those of the NIHR, the NHS or the Department of Health.

\section{References}

1. Chimen M, Kennedy A, Nirantharakumar K, Pang $T T$, Andrews R, Narendran $P$. What are the health benefits of physical activity in type 1 diabetes mellitus? A literature review. Diabetologia 2012;55:542-51. https://doi.org/10.1007/s00125-011-2403-2

2. Narendran P, Solomon TP, Kennedy A, Chimen M, Andrews RC. The time has come to test the beta cell preserving effects of exercise in patients with new onset type 1 diabetes. Diabetologia 2015;58:10-18. https://doi.org/10.1007/s00125-014-3412-8

3. Lascar N, Kennedy A, Jackson N, et al. Exercise to preserve beta cell func- tion in recent-onset type 1 diabetes mellitus (EXTOD): a study protocol for a pilot randomized controlled trial. Trials 2013;14:180. https://doi.org/10.1186/1745-6215-14-180

4. Narendran P, Jackson N, Daley A, et al. Exercise to preserve $\beta$-cell function in recent-onset type 1 diabetes mellitus (EXTOD): a randomized controlled pilot trial. Diabet Med 2017;34:1521-31. https://doi.org/10.1111/dme.13439

5. Chetan MR, Charlton MH, Thompson C, Dias RP, Andrews RC, Narendran $\mathrm{P}$. The type 1 diabetes 'honeymoon' period is five times longer in men who exercise: a case-control study. Diabet Med 2018 Aug 17. https://doi.org/10.1111/dme.13802

6. Henshall C, Narendran P, Andrews RC, et al. Qualitative study of barriers to clinical trial retention in adults with recently diagnosed type 1 diabetes. BMJ Open 2018;8(7):e022353. https://doi.org/10.1136/bmjopen-2018-022353

7. Kennedy A, Narendran P, Andrews RC, Daley A, Greenfield SM; EXTOD Group. Attitudes and barriers to exercise in adults with a recent diagnosis of type 1 diabetes: a qualitative study of participants in the Exercise for Type 1 Diabetes (EXTOD) study. BMJ Open 2018;8(1):e017813. https://doi.org/10.1136/bmjopen-2017-017813

8. Lascar N, Kennedy A, Hancock B, et al. Attitudes and barriers to exercise in adults with type 1 diabetes (T1DM) and how best to address them: a qualitative study. PLoS One 2014;9(9):e108019. https://doi.org/10.1371/journal.pone.0108019.

9. Rich R, Thompson C, Forbes A, Andrews RC, Narendran P. Healthcare professionals have poor knowledge but high levels of confidence regarding exercise in type 1 diabetes. Poster at 2015 IDF Conference, Vancover, Canada.

10. Troughton J, Sorenson J, Doherty Y, et al. Development of an education programme to support patients with type 1 diabetes to exercise safely and effectively: the EXercising for Type One Diabetes education programme (EXTOD). Poster at Diabetes UK Conference, March 2018. Diabet Med 2018;35(S1):129.

11. American Diabetes Association. Lifestyle management: standards of medical care in diabetes-2018. Diabetes Care 2018;41(Suppl 1):S38S50. https://doi.org/10.2337/dc18-S004 